\title{
PROPOSAL FOR AN INTERPRETATION OF THE HERMITIAN THEORY OF RELATIVITY
}

\author{
S. ANTOCI
}

\begin{abstract}
The equilibrium conditions for charges and currents, apparent in exact solutions of the field equations, lead one to regard the Hermitian theory of relativity as the theory of a field endowed with two sources: electromagnetic and colour four-currents.
\end{abstract}

\section{INTRODUCTION}

The Hermitian theory of relativity dates back to 1925, when Einstein proposed it as a unitary field theory of gravitation and electromagnetism [1]. Only in 1945, after several attempts of a different kind, did Einstein return 2 to the original idea, which he subsequently pursued, together with his collaborators, in several works until his death in 1955. Difficulties were soon encountered when trying to find exact solutions of the field equations, which could allow for some glimpse into the physical content of the theory 3], while approximate calculations led to disconcerting results for the motion of charged particles [4. Both Einstein [5] and Schrödinger [6] did not believe that the evidence brought against the theory by the approximate calculations could be considered really conclusive: to them, the problem of identification of the geometrical objects of the theory with physical entities was still an open one, since no special exact solution, which suggested an application to anything that might interest us, had yet been found. However, with a turn motivated more by psychological than by rational reasons, the interest in that theory faded, and the Hermitian theory of relativity is considered by present-day physicists, with a few exceptions, a subject of purely historical interest.

In recent times a method was found [7, which allows one to build solutions of the field equations of Hermitian relativity depending on three coordinates; particular solutions obtained by this method appear to have direct physical meaning if singular sources, not contemplated by the original, tentative interpretation of Einstein and Schrödinger, but called in by the very structure of the solutions, are allowed. The evidence brought by these solutions on the merit of introducing singular sources is so compelling that a thorough reexamination of the Hermitian extension of general relativity, in order to fully appreciate its physical content, is urgently required.

General Relativity and Gravitation, 19, 665 (1987). 


\section{The FIELD EQUATIONS}

By extending into the complex domain the symmetry postulates of general relativity, let us consider the Hermitian fundamental form $g_{i k}=g_{(i k)}+g_{[i k]}$ and the affine connection $\Gamma_{k l}^{i}=\Gamma_{(k l)}^{i}+\Gamma_{[k l]}^{i}$, Hermitian with respect to the lower indices; both entities depend on the real coordinates $x^{i}$, with $i$ running from 1 to 4 . We define also the contravariant tensor $g^{i k}$ by the relation

$$
g^{i l} g_{k l}=\delta_{k}^{i}
$$

and the contravariant tensor density $\mathbf{g}^{i k}=(-g)^{1 / 2} g^{i k}$, where $g=\operatorname{det}\left(g_{i k}\right)$.

Then the field equations of the Hermitian theory of relativity 8 can be written as

$$
\begin{aligned}
g_{i k, l}-g_{n k} \Gamma_{i l}^{n}-g_{i n} \Gamma_{l k}^{n} & =0, \\
\mathbf{g}^{[i s]} & =0, \\
R_{(i k)}(\Gamma) & =0, \\
R_{[i k], l}(\Gamma)+R_{[k l], i}(\Gamma)+R_{[l i], k}(\Gamma) & =0 ;
\end{aligned}
$$

$R_{i k}(\Gamma)$ is the Ricci tensor

$$
R_{i k}(\Gamma)=\Gamma_{i k, a}^{a}-\Gamma_{i a, k}^{a}-\Gamma_{i b}^{a} \Gamma_{a k}^{b}+\Gamma_{i k}^{a} \Gamma_{a b}^{b} .
$$

Let $\varepsilon^{i k l m}$ represent Levi Civita's tensor density; from $R_{[i k]}$ we can define the dual tensor density

$$
{ }^{*} \mathbf{R}^{i k}=\frac{1}{2} \varepsilon^{i k l m} R_{[l m]},
$$

and write (5) as

$$
{ }^{*} \mathbf{R}_{, s}^{[i s]}=0
$$

\section{The PRoblem of EXTERnAl SOURCES}

Before looking for particular solutions of the field equations (21)-(15), let us remember the issue whether, in the Hermitian theory of relativity, field singularities are to be allowed or not.

Einstein and Schrödinger expressed clearly their opinion on this point: for them, singularities should not be allowed at all; (3), for instance, needs to be satisfied everywhere. A comparison with Maxwell's theory then suggests that this equation is interpreted as expressing the vanishing of the magnetic current density; if this is accepted, the expression of the electric current density [5] should be $\mathbf{J}^{m}=\frac{1}{6} \varepsilon^{i k l m} g_{[[i k], l]}$. However, no static spherically symmetric solution that complies with such a proposal can exist 9, and the approximate solution of the problem of motion, done according to the above-mentioned identification, found no evidence for the Lorentz force 4].

It was Treder who proved [10 the existence of nongravitational forces in the Hermitian theory of relativity; his result (a Coulomb-like force plus a force independent of distance) could only be obtained by allowing for 
singular sources at the right-hand-side of (5). Since, in Treder's approximate calculation, the constant force cannot be made to vanish without cancelling the Coulomb-like force itself, and since constant forces were not considered in 1957 to be physically meaningful, that finding seemed to provide arguments against Hermitian relativity.

In 1980 Treder himself showed 11] that his earlier finding could be given a chromodynamic interpretation. Stimulated by this result, I propose to allow for singular sources on the right-hand-sides of both (3) and (15). These sources are conserved four-currents, and particular solutions obtained by the method of the next section show that it is indeed worth allowing for them, since a physical interpretation then becomes apparent.

\section{A METHOD FOR Finding SOLUTIONS}

Despite the complexity of the field equations (2)-(15), a simple method exists [7], that allows one to build solutions of the Hermitian theory of relativity from known vacuum solutions of the general theory of relativity. To quote this result, I assume henceforth that Latin indices run from 1 to 4, while Greek indices run from 1 to 3 .

Let $h_{i k}$ be the fundamental form of a vacuum solution of general relativity, which depends only on the three co-ordinates $x^{\lambda}$, and for which $h_{\lambda 4}=0$; we define also a purely imaginary tensor $a_{i k}=a_{i k}\left(x^{\lambda}\right)$, and we assume that its only nonvanishing components are $a_{\mu 4}=-a_{4 \mu}$. We form the tensor

$$
\alpha_{i}^{k}=a_{i l} h^{k l}=-\alpha_{i}^{k},
$$

where $h^{i k}$ is the inverse of $h_{i k}$, and we define the Hermitian fundamental form $g_{i k}$ as follows:

$$
\begin{array}{r}
g_{\lambda \mu}=h_{\lambda \mu}, \\
g_{4 \mu}=\alpha_{4}{ }^{\nu} h_{\mu \nu}, \\
g_{44}=h_{44}-\alpha_{4}{ }^{\mu} \alpha_{4}{ }^{\nu} h_{\mu \nu} .
\end{array}
$$

With such a choice of the fundamental tensor, (3) reduces to the single equation

$$
\left[\left(-\operatorname{det} h_{i k}\right)^{1 / 2} \alpha_{4}^{\lambda} h^{44}\right]_{, \lambda}=0 .
$$

In the Hermitian theory of relativity, (2) defines the affinity $\Gamma_{k l}^{i}$ in terms of the fundamental tensor; it can be uniquely solved [12] under very general circumstances for $g_{i k}$. We now solve (2) for the particular choice of the fundamental tensor given by (10); it turns out that, if $\alpha_{i}{ }^{k}$ obeys the three equations

$$
\alpha^{4}{ }_{\mu, \lambda}-\alpha_{\lambda, \mu}^{4}=0
$$


the result simplifies considerably, and the nonzero components of $\Gamma_{k l}^{i}$ can be written as

$$
\begin{aligned}
& \Gamma_{(\mu \nu)}^{\lambda}=\left\{\begin{array}{c}
\lambda \\
\mu \nu
\end{array}\right\}, \\
& \Gamma_{[4 \nu]}^{\lambda}=\alpha_{4, \nu}^{\lambda}-\left\{\begin{array}{c}
4 \\
4 \nu
\end{array}\right\} \alpha_{4}^{\lambda}+\left\{\begin{array}{c}
\lambda \\
\rho \nu
\end{array}\right\} \alpha_{4}{ }^{\varrho}, \\
& \Gamma_{(4 \nu)}^{4}=\left\{\begin{array}{c}
4 \\
4 \nu
\end{array}\right\}, \\
& \Gamma_{44}^{\lambda}=\left\{\begin{array}{c}
\lambda \\
44
\end{array}\right\}-\alpha_{4}^{\nu}\left(\Gamma_{[4 \nu]}^{\lambda}-\alpha_{4}^{\lambda} \Gamma_{(4 \nu)}^{4}\right) ;
\end{aligned}
$$

in (13),$\left\{\begin{array}{c}i \\ k\end{array}\right\}$ is the Christoffel connection built with $h_{i k}$. We are now in a position to write the Ricci tensor $R_{i k}(\Gamma)$, and we find that, if (11) and (12) hold, its nonvanishing components read

$$
\begin{array}{r}
R_{\lambda \mu}=S_{\lambda \mu}, \\
R_{4 \mu}=\alpha_{4}{ }^{\nu} S_{\mu \nu}+\left(\alpha_{4}{ }^{\nu}\left\{\begin{array}{c}
4 \\
4 \nu
\end{array}\right)_{, \mu},\right. \\
R_{44}=S_{44}-\alpha_{4}{ }^{\mu} \alpha_{4}{ }^{\nu} S_{\mu \nu},
\end{array}
$$

where $S_{i k}$ is the Ricci tensor built with $\left\{\begin{array}{c}i \\ k l\end{array}\right\}$. But $S_{i k}=0$ for a vacuum solution of general relativity; therefore, even the field equations (4) and (5) of the Hermitian theory are satisfied. Under the circumstances mentioned above, the problem of solving (2)-(15) reduces to a simpler task: to satisfy (11) and (12) for a given field $h_{i k}$, solution of general relativity. Such a task is immediately accomplished when $h_{i k}$ corresponds to some simple form of the Minkowski metric.

\section{Electrostatics}

We can, for instance, start with $h_{i k}$ as representing the Minkowski metric referred to Cartesian coordinates $x, y, z, t$ and obtain the solution

$$
g_{i k}=\left(\begin{array}{rrrr}
-1 & 0 & 0 & a \\
0 & -1 & 0 & b \\
0 & 0 & -1 & c \\
-a & -b & -c & d
\end{array}\right)
$$

with

$$
d=1+a^{2}+b^{2}+c^{2}
$$

and

$$
a=i \chi_{, x}, b=i \chi_{, y}, c=i \chi_{, z}, \quad \chi_{, x x}+\chi_{, y y}+\chi_{, z z}=0 .
$$

The imaginary part $g_{[i k]}$ of this solution just looks like the general electrostatic solution of Maxwell's theory. If we allow for singularities in (3), the electrostatic field due to $n$ point charges $h_{q}$, located at $x=x_{q}, y=y_{q}$, $z=z_{q}$, can be built by taking

$$
\chi=-\sum_{q=1}^{n} \frac{h_{q}}{p_{q}},
$$


where

$$
p_{q}=\left[\left(x-x_{q}\right)^{2}+\left(y-y_{q}\right)^{2}+\left(z-z_{q}\right)^{2}\right]^{1 / 2} .
$$

But a nonlinear theory like ours should give more information than Maxwell's equations: it should predict also the equations of motion, i.e., in this case, the conditions for the electrostatic equilibrium of charges $h_{q}$. We have agreed to represent charges by singularities, and previous experience with the problem of motion in general relativity has shown [13] that the shape of the singularities needs to be restricted in order to get a meaningful result. We can learn from the solution what kind of restriction must be imposed; we expect that such a restriction may arise from terms which are nonlinear in charges $h_{q}$. Let us consider the only nonlinear term which appears in $g_{i k}$ :

$$
d=1-\sum_{q=1}^{n} \frac{h_{q}^{2}}{p_{q}^{4}}
$$

$$
-\sum_{q, q^{\prime}=1}^{n\left(q \neq q^{\prime}\right)} h_{q} h_{q^{\prime}} \frac{\left(x-x_{q}\right)\left(x-x_{q^{\prime}}\right)+\left(y-y_{q}\right)\left(y-y_{q^{\prime}}\right)+\left(z-z_{q}\right)\left(z-z_{q^{\prime}}\right)}{p_{q}^{3} p_{q^{\prime}}^{3}} .
$$

If we want to avoid that $d$ must exhibit a singularity in the form of a jump, arising from the presence of the other charges, at the position where the $q$ th charge is located, we have to require that

$$
\sum_{q^{\prime} \neq q}^{n} h_{q^{\prime}} \frac{x_{q}-x_{q^{\prime}}}{r_{q q^{\prime}}^{3}}=\sum_{q^{\prime} \neq q}^{n} h_{q^{\prime}} \frac{y_{q}-y_{q^{\prime}}}{r_{q q^{\prime}}^{3}}=\sum_{q^{\prime} \neq q}^{n} h_{q^{\prime}} \frac{z_{q}-z_{q^{\prime}}}{r_{q q^{\prime}}^{3}}=0,
$$

where $r_{q q^{\prime}}$ is the Cartesian distance between the two charges $q$ and $q^{\prime}$. Equation (21) indeed expresses the equilibrium conditions for $n$ point electric charges; when $g_{(i k)}$ is assumed to be the metric tensor of the Hermitian theory, this result is true not only in the coordinate space, but also in the physical space; the no-jump rule appears to be a physically relevant restriction on singularities.

\section{Electric CURRents in EQUilibrium}

When $h_{i k}$ is given by the Minkowski metric referred to Cartesian coordinates, we can get also another solution, which depends on $x, y$ and $t$ :

$$
g_{i k}=\left(\begin{array}{rrrr}
-1 & 0 & e & 0 \\
0 & -1 & f & 0 \\
-e & -f & h & c \\
0 & 0 & -c & 1
\end{array}\right)
$$

with

$$
h=-1+e^{2}+f^{2}-c^{2}
$$


and

$$
e=i \xi_{, x}, f=i \xi_{, y}, c=-i \xi_{, t}, \quad \xi_{, x x}+\xi_{, y y}-\xi_{, t t}=0 ;
$$

even in this case $g_{[i k]}$ obeys Maxwell's equations.

We allow for singularities in (3) and consider the particular solution for which

$$
\xi=\sum_{q=1}^{n} l_{q} \ln p_{q}
$$

where

$$
p_{q}=\left[\left(x-x_{q}\right)^{2}+\left(y-y_{q}\right)^{2}\right]^{1 / 2} .
$$

In this solution $g_{[i k]}$ represents the magnetic field generated by $n$ steady currents; each current, with intensity $l_{q}$, is running along a wire parallel to the $z$ axis, which intersects the $x, y$ plane at $x=x_{q}, y=y_{q}$. The no-jump rule, which now restricts the nonlinear term $h$, requires that

$$
\sum_{q^{\prime} \neq q}^{n} l_{q^{\prime}} \frac{x_{q}-x_{q^{\prime}}}{r_{q q^{\prime}}^{2}}=\sum_{q^{\prime} \neq q}^{n} l_{q^{\prime}} \frac{y_{q}-y_{q^{\prime}}}{r_{q q^{\prime}}^{2}}=0,
$$

where $r_{q q^{\prime}}$ is the Cartesian distance between the two wires $q$ and $q^{\prime}$. Equation (27), as expected, predicts the equilibrium positions of $n$ parallel wires at rest, run by steady currents.

As a further example, we can take

$$
\begin{array}{r}
\xi=\sum_{q=1}^{n} \frac{l_{q}}{p_{q}}, \\
p_{q}=\left[\left(t-t_{q}\right)^{2}-\left(x-x_{q}\right)^{2}-\left(y-y_{q}\right)^{2}\right]^{1 / 2} .
\end{array}
$$

In the sum each term is considered only if

$$
\left(t-t_{q}\right)^{2}>\left(x-x_{q}\right)^{2}+\left(y-y_{q}\right)^{2} .
$$

In such a solution $g_{[i k]}$ represents the superposition of cylindrical electromagnetic waves radiated by $n$ current pulses, occurring in wires parallel to the $z$ axis; the $q$ th pulse occurs at time $t=t_{q}$ on the wire which intersects the $x, y$ plane at $x=x_{q}, y=y_{q}$; both advanced and retarded fields with equal weight are taken. The no-jump condition, when applied to the singularities of this solution, prescribes that

$$
\sum_{q^{\prime} \neq q}^{n} l_{q^{\prime}} \frac{t_{q}-t_{q^{\prime}}}{s_{q q^{\prime}}^{3}}=\sum_{q^{\prime} \neq q}^{n} l_{q^{\prime}} \frac{x_{q}-x_{q^{\prime}}}{s_{q q^{\prime}}^{3}}=\sum_{q^{\prime} \neq q}^{n} l_{q^{\prime}} \frac{y_{q}-y_{q^{\prime}}}{s_{q q^{\prime}}^{3}}=0,
$$

where

$$
s_{q q^{\prime}}=\left[\left(t_{q}-t_{q^{\prime}}\right)^{2}-\left(x_{q}-x_{q^{\prime}}\right)^{2}-\left(y_{q}-y_{q^{\prime}}\right)^{2}\right]^{1 / 2} .
$$

Again, a physically correct equilibrium condition, in agreement with electromagnetism, is found. When (30) holds, the electromagnetic field due to 
the other current pulses vanishes at the time and place where the $q$ th current pulse is occurring; no allowance is made for exchanges of energy and momentum between the current pulses.

\section{Electric CHARges IN UNIFORMLY ACCELERATED MOtion}

It would be interesting to provide an example of a solution allowing for exchanges of energy and momentum which were absent in the solutions considered up to now; as everybody knows, such exchanges occur when an electric charge is accelerating under the influence of an electromagnetic field. One such example can be found if we take for $h_{i k}$ the form

$$
h_{i k}=\left(\begin{array}{rrrr}
-1 & 0 & 0 & 0 \\
0 & -1 & 0 & 0 \\
0 & 0 & -1 & 0 \\
0 & 0 & 0 & z^{2}
\end{array}\right)
$$

referred to Cartesian coordinates $x, y, z, t$, and we build the solution

$$
g_{i k}=\left(\begin{array}{rrrr}
-1 & 0 & 0 & a \\
0 & -1 & 0 & b \\
0 & 0 & -1 & c \\
-a & -b & -c & d
\end{array}\right)
$$

with

$$
d=z^{2}+a^{2}+b^{2}+c^{2}
$$

and

$$
a=i z^{2} \chi_{, x}, b=i z^{2} \chi_{, y}, c=i z^{2} \chi_{, z}, \quad \chi_{, x x}+\chi_{, y y}+\chi_{, z z}+\left(\chi_{, z} / z\right)=0 .
$$

We allow for singularities in (3) and consider the particular solution with

$$
\chi=-\sum_{q=1}^{n} \frac{K_{q}}{p_{q}}+m \ln z,
$$

where

$$
\begin{array}{r}
p_{q}=\left[\left(s+h_{q}\right)^{2}-2 h_{q} z^{2}\right]^{1 / 2}, \\
s=\frac{1}{2}\left(x^{2}+y^{2}+z^{2}\right) ;
\end{array}
$$


$K_{q}, h_{q}$ and $m$ are constants; we assume $h_{q}>0$. We get

$$
\begin{array}{r}
a=i \sum_{q=1}^{n} \frac{K_{q} z^{2} x\left(s+h_{q}\right)}{p_{q}^{3}}, \\
b=i \sum_{q=1}^{n} \frac{K_{q} z^{2} y\left(s+h_{q}\right)}{p_{q}^{3}}, \\
c=i \sum_{q=1}^{n} \frac{K_{q} z^{3}\left(s-h_{q}\right)}{p_{q}^{3}}+i m z,
\end{array}
$$

and

$$
d=z^{2}(1-F)
$$

where

$$
\begin{aligned}
F=2 s z^{2} \sum_{q=1}^{n} \frac{K_{q}^{2}}{p_{q}^{4}}+ & m^{2}+z^{2}\left(x^{2}+y^{2}\right) \sum_{q, q^{\prime}=1}^{n\left(q \neq q^{\prime}\right)} \frac{K_{q} K_{q^{\prime}}\left(s+h_{q}\right)\left(s+h_{q^{\prime}}\right)}{p_{q}^{3} p_{q^{\prime}}^{3}} \\
& +z^{4} \sum_{q=1}^{n} \frac{K_{q}\left(s-h_{q}\right)}{p_{q}^{3}}\left(\frac{2 m}{z^{2}}+\sum_{q^{\prime} \neq q}^{n} \frac{K_{q^{\prime}}\left(s-h_{q^{\prime}}\right)}{p_{q^{\prime}}^{3}}\right) .
\end{aligned}
$$

To understand the meaning of this solution, let us perform the coordinate transformation

$$
r^{\prime}=\left(x^{2}+y^{2}\right)^{1 / 2}, z^{\prime}=z \cosh t, \varphi^{\prime}=\arctan (y / x), t^{\prime}=z \sinh t,
$$

and look at the transformed tensor $g_{i k}^{\prime}$. Its nonzero components are:

$$
\begin{array}{r}
g_{11}^{\prime}=-1, \\
g_{21}^{\prime}=i \sum_{q=1}^{n} \frac{K_{q} r^{\prime} t^{\prime}\left(s^{\prime}+h_{q}\right)}{p_{q}^{\prime 3}}, \\
g_{41}^{\prime}=-i \sum_{q=1}^{n} \frac{K_{q} r^{\prime} z^{\prime}\left(s^{\prime}+h_{q}\right)}{p_{q}^{\prime 3}}, \\
g_{22}^{\prime}=-1-\frac{t^{\prime 2}}{z^{\prime 2}-t^{\prime 2}} F^{\prime}, \\
\frac{z^{\prime} t^{\prime}}{z^{\prime 2}-t^{\prime 2}} F^{\prime}-i \sum_{q=1}^{n} \frac{K_{q}\left(z^{\prime 2}-t^{\prime 2}\right)\left(s^{\prime}-h_{q}\right)}{p_{q}^{\prime 3}}-i m, \\
g_{44}^{\prime}=1-\frac{z^{\prime 2}}{z^{\prime 2}-t^{\prime 2}} F^{\prime} .
\end{array}
$$

In (42), $s^{\prime}, p_{q}^{\prime}, F^{\prime}$ are those functions of the primed variables $r^{\prime}, z^{\prime}, t^{\prime}$ which are obtained from $s, p_{q}, F$ when the change of variables is performed in them. 
The form (42) does not hold as a solution only when $z^{\prime 2}>t^{\prime 2}$, as it would appear from the coordinate transformation given by (41); it is valid in the whole primed space-time. This fact can be readily ascertained by building from the metric

$$
h_{i k}=\left(\begin{array}{rrrr}
-1 & 0 & 0 & 0 \\
0 & -1 & 0 & 0 \\
0 & 0 & -t^{2} & 0 \\
0 & 0 & 0 & 1
\end{array}\right),
$$

referred to Cartesian coordinates $x, y, z, t$, a Hermitian solution which, under the coordinate transformation

$$
r^{\prime}=\left(x^{2}+y^{2}\right)^{1 / 2}, z^{\prime}=t \sinh z, \varphi^{\prime}=\arctan (y / x), t^{\prime}=t \cosh z,
$$

reduces to the form (42) for $z^{\prime 2}<t^{\prime 2}$; the two halves join smoothly along the hypersurfaces $z^{\prime 2}=t^{\prime 2}$.

The solution displays essential singularities only at the points for which

$$
r^{\prime}=0, z^{\prime 2}-t^{\prime 2}=2 h_{q}, q=1, \ldots, n .
$$

Let us evaluate the integral

$$
I=\frac{1}{8 \pi i} \int_{\Sigma} \mathbf{g}^{[i k]} \mathrm{d} f_{i k}^{*}
$$

where $\mathrm{d} f_{i k}^{*}$ is the surface element of an ordinary surface $\Sigma$ which envelops one of the singularities; we find $I=K_{q}$. We can interpret this solution as describing the field of $2 n$ point electric charges $K_{q}$ which perform accelerated motions along the $z^{\prime}$ axis in the presence of a constant electric field, with intensity $m$, directed along the same axis. Each charge performs a hyperbolic motion, with acceleration $a= \pm\left(2 h_{q}\right)^{-1 / 2}$, with respect to the coordinate space; in the limit, when both the charges and the external field tend to vanishing values, the motion results to be hyperbolic even in the physical space. The motion of $n$ of these charges occurs entirely in the half-space with $z^{\prime}>0$, and it is the mirror image of the motion of the other $n$ charges, with respect to the plane $z^{\prime}=0$.

We can restrict the analysis to what goes on in the half-space with $z^{\prime}>0$; in fact, due to their peculiar motion, the charges which are in the halfspace with $z^{\prime}>0$ do not interact with the charges which are in the other half-space. According to the no-jump rule, which in this case restricts the nonlinear term $F^{\prime}$, only those motions are allowed for which

$$
K_{q} m+\sum_{q^{\prime} \neq q}^{n} \frac{2 K_{q} K_{q^{\prime}} h_{q^{\prime}}\left(h_{q}-h_{q^{\prime}}\right)}{\left|h_{q}-h_{q^{\prime}}\right|^{3}}+\sum_{q^{\prime} \neq q}^{n} \frac{2 K_{q} K_{q^{\prime}}}{\left|h_{q}-h_{q^{\prime}}\right|}=0 .
$$

This regularity condition can be interpreted physically as an equilibrium condition for the forces acting ot the $q$ th charge. We recognize, in the first term of (47), the force exerted by the constant electric field with intensity $m$. The second term is the sum of the electric forces exerted by the charges $K_{q^{\prime}}$ in hyperbolic motion [14] on the charge $K_{q}$. The last term is new, and 
I interpret it as expressing the inertial force felt by the charge $K_{q}$. The existence of such a term is expected on physical grounds, since the charge $K_{q}$ must acquire inertia due to its very interaction with the other charges. Obviously, this interpretation needs to be confirmed by further examples; it would be a nice feature of the Hermitian theory if in it the existence of masses could be derived from the existence of charges, rather than postulated independently as occurs in general relativity.

\section{Colour sources}

Up to now, only solutions with singular sources at the right-hand side of (31) have been considered; they can be named electromagnetic. But we can also allow for singular sources in (8), the equivalent of field equation (5). Both (3) and (8), in fact, allow for the addition of a conserved four-current at the right-hand side.

We can find a solution with this behaviour if we start from the Minkowski metric $h_{i k}$ referred to polar cylindrical coordinates $r, z, \varphi, t$ :

$$
h_{i k}=\left(\begin{array}{rrrr}
-1 & 0 & 0 & 0 \\
0 & -1 & 0 & 0 \\
0 & 0 & -r^{2} & 0 \\
0 & 0 & 0 & 1
\end{array}\right) .
$$

We get the solution 15

$$
g_{i k}=\left(\begin{array}{rrrr}
-1 & 0 & \delta & 0 \\
0 & -1 & \varepsilon & 0 \\
-\delta & -\varepsilon & \zeta & \tau \\
0 & 0 & -\tau & 1
\end{array}\right)
$$

with

$$
\zeta=-r^{2}+\delta^{2}+\varepsilon^{2}-\tau^{2}
$$

and

$$
\delta=i r^{2} \psi_{, r}, \varepsilon=i r^{2} \psi_{, z}, \tau=-i r^{2} \psi_{, t}, \quad \psi_{, r r}+\left(\psi_{, r} / r\right)+\psi_{, z z}-\psi_{, t t}=0
$$

Let us consider the particular solution for which

$$
\psi=-\sum_{q=1}^{n} K_{q} \ln \frac{p_{q}+z-z_{q}}{r}+m \ln r,
$$

where

$$
p_{q}=\left[r^{2}+\left(z-z_{q}\right)^{2}\right]^{1 / 2}
$$


$K_{q}, z_{q}$ and $m$ are constants. We get

$$
\begin{array}{r}
\delta=i \sum_{q=1}^{n} \frac{K_{q} r\left(z-z_{q}\right)}{p_{q}}+i m r \\
\varepsilon=-i \sum_{q=1}^{n} \frac{K_{q} r^{2}}{p_{q}}, \tau=0,
\end{array}
$$

and

$$
\zeta=-r^{2}(1+F)
$$

where

$$
\begin{array}{r}
F=\sum_{q=1}^{n} K_{q}^{2}+m^{2}+r^{2} \sum_{q, q^{\prime}=1}^{n\left(q \neq q^{\prime}\right)} \frac{K_{q} K_{q^{\prime}}}{p_{q} p_{q^{\prime}}} \\
+\sum_{q=1}^{n} \frac{K_{q}\left(z-z_{q}\right)}{p_{q}}\left[2 m+\sum_{q^{\prime} \neq q}^{n} \frac{K_{q^{\prime}}\left(z-z_{q^{\prime}}\right)}{p_{q^{\prime}}}\right] .
\end{array}
$$

We go over to Cartesian coordinates with the transformation

$$
x^{\prime}=r \cos \varphi, y^{\prime}=r \sin \varphi, z^{\prime}=z, t^{\prime}=t ;
$$

the nonzero components of the transformed tensor $g_{i k}^{\prime}$ read

$$
\begin{array}{r}
g_{11}^{\prime}=-1-\frac{y^{\prime 2}}{x^{\prime 2}+y^{\prime 2}} F^{\prime}, \\
g_{21}^{\prime}=\frac{x^{\prime} y^{\prime}}{x^{\prime 2}+y^{\prime 2}} F^{\prime}-i \sum_{q=1}^{n} \frac{K_{q}\left(z^{\prime}-z_{q}\right)}{p^{\prime}{ }_{q}}-i m, \\
g_{31}^{\prime}=i \sum_{q=1}^{n} \frac{K_{q} y^{\prime}}{p^{\prime}}, \\
g_{22}^{\prime}=-1-\frac{x^{\prime 2}}{x^{\prime 2}+y^{\prime 2}} F^{\prime}, \\
g_{32}^{\prime}=-i \sum_{q=1}^{n} \frac{K_{q} x^{\prime}}{p^{\prime}{ }_{q}}, \\
g_{33}^{\prime}=-1, \\
g_{44}^{\prime}=1 .
\end{array}
$$

$p_{q}^{\prime}$ and $F^{\prime}$ are those functions of $x^{\prime}, y^{\prime}, z^{\prime}$ which can be obtained from $p_{q}$ and $F$ through the change of variables. The solution displays essential singularities at

$$
x^{\prime}=y^{\prime}=0, z^{\prime}=z_{q}, q=1, \ldots, n .
$$


Let us evaluate the integral

$$
I=-\frac{1}{8 \pi i} \int_{\Sigma} R_{[i k]} \mathrm{d} f^{i k}
$$

extended to an ordinary surface $\Sigma$ which envelops one of the singularities. We get $I=K_{q}$. It seems therefore that we have to do with $n$ charges of a new kind, at rest on the $z^{\prime}$ axis, in the presence of a constant magnetic field of intensity $m$, directed along the same axis. The no-jump rule for singularities yields the equilibrium condition

$$
\sum_{q^{\prime} \neq q}^{n} \frac{K_{q} K_{q^{\prime}}\left(z_{q}-z_{q^{\prime}}\right)}{\left|z_{q}-z_{q^{\prime}}\right|}+K_{q} m=0
$$

which we interpret as expressing a force balance. The first term of (61) shows that the new charges interact mutually with forces which do not depend on the distance between them; this occurs in the coordinate space and even in the physical space if $g_{(i k)}$ is the metric tensor. As was mentioned in Section 3 , such forces were found in 1957 by Treder with an approximate calculation 10] and were recently interpreted [1] by the same author as confining forces between colour charges. The second term of (61) shows that the colour charges predicted by the Hermitian theory of relativity, when sitting at rest in a constant magnetic field, feel a constant pull, as magnetic monopoles would do.

This result does not mean that the new charges are magnetic monopoles; in fact, the field $g_{[i k]}$ does not obey Maxwell's equations, and we have just shown that the charges $K_{q}$ cannot exist in a free state, since they are bound by constant forces. A mixed behaviour of this kind is at variance with the predictions of quantum chromodynamics, but we cannot conclude from here that it does not agree with experience. Of course, such new behaviour can be expected from a truly unified theory, in which all the fields merge in a single entity [6] without artificial boundaries between them.

\section{Conclusion}

If allowance is made for singular, conserved sources at the right-hand sides of (3) and (8), then particular exact solutions to the field equations of the Hermitian theory of relativity find a direct physical interpretation. These solutions show that past negative results found with this theory cannot be imputed to the field equations per se, but only to a false identification of the physical entities with the geometrical objects of the theory. With a new identification, the outcome of Einstein's lifelong effort to achieve a unified field theory no longer appears to be a failure, as is currently believed. It is indeed worth investigating again how far the Hermitian theory of relativity can go in accounting for gravitational, electromagnetic and nuclear phenomena. 
Aknowledgments. I am indebted to Professor E. Kreisel and Professor H.-J. Treder for many helpful discussions and for the support given to my work.

\section{REFERENCES}

1. Einstein, A. (1925). S. B. Preuss. Akad. Wiss., 22, 414.

2. Einstein, A. (1945). Ann. Math., 46, 578.

3. Papapetrou, A. (1948). Proc. R. I. A. A52, 69; Wyman, M. (1950). Can. J. Math., 2, 427.

4. Callaway, J. (1953). Phys. Rev., 92, 1567.

5. Einstein, A. (1955). The Meaning of Relativity (Princeton University Press, Princeton).

6. Schrödinger, E. (1954). Space-Time Structure. (Cambridge University Press, Cambridge).

7. Antoci, S. (1987). Ann. Phys. (Leipzig), 44, 297.

Also: http://arxiv.org/abs/gr-qc/0108042

8. Einstein, A. (1948). Rev. Mod. Phys., 20, 35.

9. Tiwari, R. and Pant, D.N. (1970). Phys. Lett., A33, 505.

10. Treder, H.-J. (1957). Ann. Phys. (Leipzig), 19, 369.

11. Treder, H.-J. (1980). Ann. Phys. (Leipzig), 37, 250; (1983). ibidem, 40, 81.

12. Tonnelat, M. A. (1955). La Théorie du Champ Unifié d'Einstein (Gauthier-Villars, Paris); Hlavatý, V. (1957). Geometry of Einstein's Unified Field Theory (Noordhoff, Groningen).

13. Einstein, A. and Infeld, L. (1949). Can. J. Math., 1, 209.

14. Born, M. (1909). Ann. Phys. (Leipzig), 30, 1; Fulton, T. and Rohrlich, F. (1960). Ann. Phys. (N.Y.), 9, 499.

15. Antoci, S. (1984). Ann. Phys. (Leipzig), 41, 419.

Dipartimento di Fisica “A. Volta”, Via A. Bassi, 6, 27100 Pavia, Italy. 